\title{
LETTERS
}

\section{Dry powder inhalers are environmentally preferable to metered-dose inhalers}

In their CMAJ commentary, Drs. Beauchesne and Lemiere outlined the key practice changes recommended in the 2019 Global Initiative for Asthma report. ${ }^{1}$

As they noted, available evidence supports a pragmatic approach to inhaler therapy for patients older than 12 years with mild asthma: the as-needed use of the budesonide-formoterol combination inhaler.

This shift could also be good for the environment.

Metered-dose inhalers are an important contributor to health care's climate footprint. They contain high levels of hydrofluorocarbons that, when released into the atmosphere, act as potent greenhouse gases. Metered-dose inhalers are estimated to contribute $3.1 \%$ of the carbon footprint of the National Health Service in the United Kingdom ${ }^{2}$ and roughly $0.03 \%$ of annual global greenhouse gas emissions. ${ }^{3}$ Dry powder inhalers are clinically appropriate and cost-effective alternatives. ${ }^{4,5}$ In the UK, the National Institute of Health and Care Excellence (NICE) has developed an evidence-based decision aid for patients with asthma, which includes information about environmental impact, to assist patients and health care professionals in selecting the most suitable inhaler device (www.nice.org.uk/guidance/ng80/resources/ inhalers-for-asthma-patient-decision-aid-pdf -6727144573).

As Beauchesne and Lemiere stated, "budesonide-formoterol is the only inhaler that has received approval from Health Canada as an anti-inflammatory reliever therapy in patients at least 12 years of age with mild, moderate and severe asthma." We point out that this product is available only as a dry powder inhaler. Thus, increased uptake of the Global Initiative for Asthma recommendations could contribute to our fight against climate change.

For meaningful impact in Canada, however, dry powder inhaler versions of asthma medications need to be covered by provincial and territorial drug benefit programs. This is often not the case.

We urge provincial and territorial drug benefit programs to fund the budesonideformoterol combination immediately. We also suggest that the Canadian Agency for Drugs and Technologies in Health - Canada's version of NICE - support the transition with accessible information to engage patients and clinicians in conversations about "environmentally preferable" asthma therapy. This will lead to better asthma control for the population, lower long-term costs and decreased greenhouse gas emissions.

\section{Kimberly Wintemute MD}

Family physician, Department of Family and Community Medicine, University of Toronto, Toronto, Ont.

\section{Fiona Miller PhD}

Professor of Health Policy; director, Centre for Sustainable Health Systems, Institute of Health Policy, Management and Evaluation, University of Toronto, Toronto, Ont.

\section{References}

1. Beauchesne MF, Lemiere C. Global Initiative for Asthma report: How will new recommendations affect practice in Canada? CMAJ 2020;192: E456-8.

2. vanHove M, Leng G. A more sustainable NHS. BMJ 2019;366:14930.

3. UNEP 2014 report of the Medical Technical Options Committee. Nairobi [Kenya]: United Nations Environment Programme; 2015. Available: http://conf. montreal-protocol.org/meeting/oewg/oewg-36/ presession/Background\%20Documents\%20are \%20available\%20in\%20English\%20only/MTOC -Assessment-Report-2014.pdf (accessed 2020 July 3).

4. Wilkinson AJ, Braggins R, Steinbach I, et al. Costs of switching to low global warming potential inhalers. An economic and carbon footprint analysis of NHS prescription data in England. BMJ Open 2019;9:e028763.

5. Janson C, Henderson R, Löfdahl M, et al. Carbon footprint impact of the choice of inhalers for asthma and COPD. Thorax 2020;75:82-4.

— Cite as: CMAJ 2020 July 8;192:E846. doi: 10.1503/cmaj.75949

Competing interests: None declared. 